\title{
Effects of the Weak Polar Fields of Solar Cycle 23: Investigation Using OMNI for the STEREO Mission Period
}

\author{
C.O. Lee $\cdot$ J.G. Luhmann $\cdot$ X.P. Zhao $\cdot$ Y. Liu $\cdot$ P. Riley $\cdot$ \\ C.N. Arge • C.T. Russell • I. de Pater
}

Received: 7 December 2008 / Accepted: 25 March 2009 / Published online: 9 April 2009

(C) The Author(s) 2009. This article is published with open access at Springerlink.com

\begin{abstract}
The current solar cycle minimum seems to have unusual properties that appear to be related to weak solar polar magnetic fields. We investigate signatures of this unusual polar
\end{abstract}

STEREO Science Results at Solar Minimum

Guest Editors: Eric R. Christian, Michael L. Kaiser, Therese A. Kucera, O.C. St. Cyr.

C.O. Lee $(\varangle) \cdot$ J.G. Luhmann

Space Sciences Laboratory, University of California, Berkeley, CA, USA

e-mail: clee@ssl.berkeley.edu

J.G. Luhmann

e-mail: jgluhman@ssl.berkeley.edu

C.O. Lee $\cdot$ I. de Pater

Department of Earth and Planetary Science, University of California, Berkeley, CA, USA

I. de Pater

e-mail: imke@astron.berkeley.edu

X.P. Zhao · Y. Liu

W. W. Hansen Experimental Physics Laboratory, Stanford University, Stanford, CA, USA

X.P. Zhao

e-mail: xpzhao@solar.stanford.edu

Y. Liu

e-mail: yliu@solar.stanford.edu

P. Riley

Predictive Science, Inc., San Diego, CA, USA

e-mail: pete@predsci.com

C.N. Arge

Air Force Research Laboratory/Space Vehicles Directorate, Kirtland Air Force Base, NM, USA

e-mail: nick.arge@kirtland.af.mil

C.T. Russell

Institute of Geophysics and Planetary Physics, University of California, Los Angeles, CA, USA

e-mail: ctrussell@igpp.ucla.edu 
field in the ecliptic near-Earth interplanetary magnetic field (IMF) for the STEREO period of observations. Using $1 \mathrm{AU}$ OMNI data, we find that for the current solar cycle declining phase to minimum period the peak of the distribution for the values of the ecliptic IMF magnitude is lower compared to a similar phase of the previous solar cycle. We investigate the sources of these weak fields. Our results suggest that they are related to the solar wind stream structure, which is enhanced by the weak polar fields. The direct role of the solar field is therefore complicated by this effect, which redistributes the solar magnetic flux at 1 AU nonuniformly at low to mid heliolatitudes.

Keywords STEREO mission · Solar wind · Solar cycle, models · Solar cycle, observations · Magnetic fields, observations · Magnetic fields, interplanetary

\section{Introduction}

The recent solar cycle 23 deep solar minimum period appears to be unlike previous ones since the dawn of the Space Age. The Sun, which has been spotless for over 200 days in the year 2008, has weaker polar magnetic fields. The solar polar field values observed using ground-based magnetographs are half their previous solar minimum values (see Figure 1). This results in changes in the interplanetary medium. Measurements from the Ulysses spacecraft (Balogh et al., 1992; Bame et al., 1992) fast-latitude scans reveal that the average radial magnetic field values observed during the current minimum period are only about two-thirds of their values measured during the previous minimum period (Smith and Balogh, 2009). The related Ulysses plasma measurements reveal that the solar wind emanating from the large polar coronal holes is slightly slower, significantly less dense, and cooler, with less mass and momentum flux than previous solar minimum values (McComas et al., 2008; Issautier et al., 2008).

In this study we examine the near-ecliptic solar wind and interplanetary magnetic field (IMF) at $1 \mathrm{AU}$ for differences, if any, that exist as a direct result of the weaker solar polar fields of this cycle. We now know much more about the solar wind in terms of its different

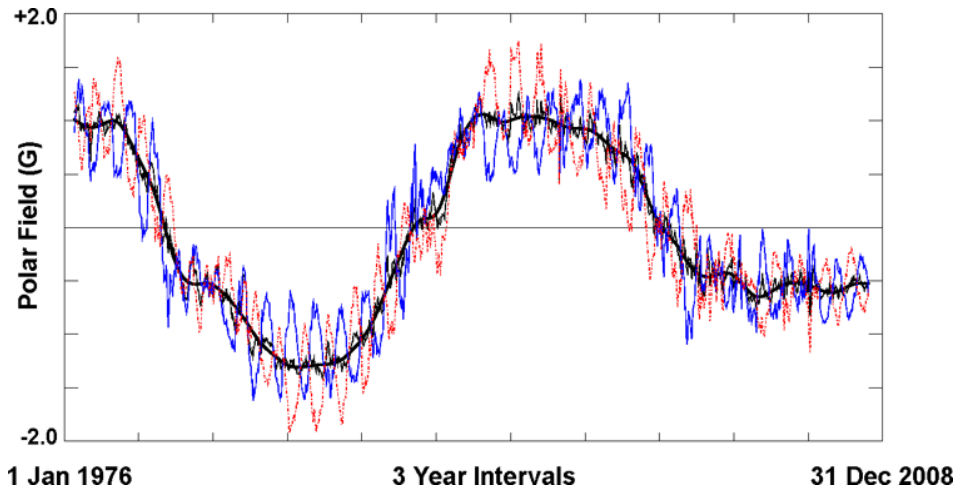

Figure 1 Solar polar field strength versus time. The figure is adapted from the original on the Stanford Wilcox Observatory Web site (http://wso.stanford.edu/gifs/Polar.gif). The blue (red) line represents the field strength observed in the north (south); the thin (thick) black line represents the average (smoothed average). Note that the calibration of the $y$-axis is not very reliable since the measurements are obtained from the poleward bins of the magnetograph (see Svalgaard, Duvall, and Scherrer, 1978, for a discussion). Nevertheless, the overall trend is shown very well. 
Figure 2 Sunspot number (SSN) for the periods of interest. The black bars indicate the period in SC 22 for 23 February to 22 November 1995. The red bars indicate the period in SC 23 for 4 February to 4 November 2007.

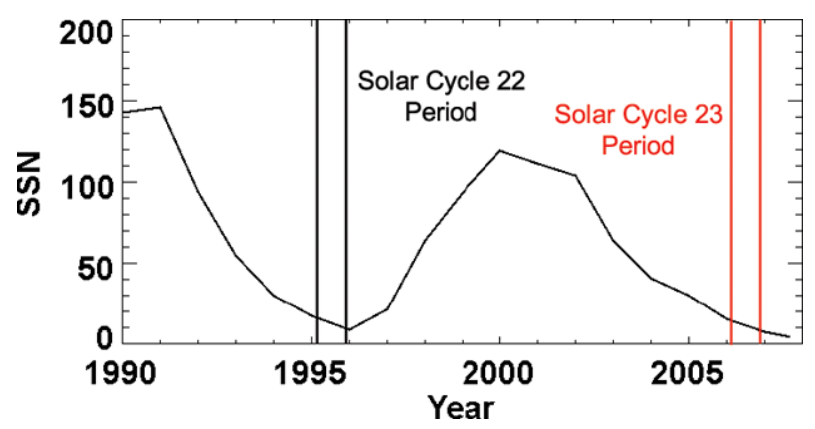

source regions. In particular, 3D models based on synoptic maps of the solar magnetic fields (e.g., Riley, Linker, and Mikic, 2001; Arge and Pizzo, 2000) have allowed us to realistically map those sources into interplanetary space for solar minimum conditions. Previous model results have shown us that the ecliptic solar wind has a complicated mixture of open field source regions consisting of polar coronal holes, low-latitude coronal holes, and polar coronal hole extensions (e.g., Luhmann et al., 2002). Luhmann et al. (2009) discuss the way in which weaker polar fields can lead to more prominent low- to mid-latitude coronal holes and therefore stronger high-speed wind streams. The results presented in this study are of particular interest for terrestrial space weather as well as the interpretation of observations from the Solar TErrestrial RElations Observatory (STEREO) mission ( $c f$. Kaiser, 2005).

The focus of our study is on the STEREO mission period. However, we use the OMNI solar wind plasma and magnetic field data to compare with a similar phase of the previous solar cycle. The OMNI hourly resolution data sets, obtained from the NASA Goddard Space Flight Center OMNIWeb Web site (http://omniweb.gsfc.nasa.gov/), have been normalized to $1 \mathrm{AU}$ and have been transformed to the RTN (radial, tangential, and normal) coordinate system. The period of observations analyzed corresponds to the heliocentric phase of the STEREO twin spacecraft.

At the time of this study, the available STEREO data ranged from 14 February to 31 October 2007 for STEREO-A and from 1 March to 31 October 2007 for STEREO-B. These time periods fall within 10 Carrington rotations (CRs), 2053 - 2062 (4 February to 4 November 2007) of the solar cycle 23 (hereafter, SC 23) late declining to solar minimum phase. We thus select the OMNI data for this time range as well as from the previous cycle, SC 22 (CRs 1893 - 1902, 23 February to 22 November 1995), when the solar cycle phase is similar and the data coverage is comparable (see Figure 2).

\section{Observations: IMF at $1 \mathrm{AU}$}

Figure 3(a) shows a histogram of density observed at 1 AU during SC 22 (black) and 23 (red). These histograms have been normalized by the total number of measurements so that they are directly comparable. The peak occurrence for the SC 22 period is centered at $3.5 \mathrm{~cm}^{-3}$. For the SC 23 period, the distribution is noticeably shifted toward lower values by about $30 \%$, with the peak of the distribution centered at $2.5 \mathrm{~cm}^{-3}$. The overall density distribution for the SC 23 period is narrower and has a percent occurrence that is much larger for values below $5 \mathrm{~cm}^{-3}$. Notice that, above $6 \mathrm{~cm}^{-3}$, the SC 22 distribution has a larger percent occurrence. 

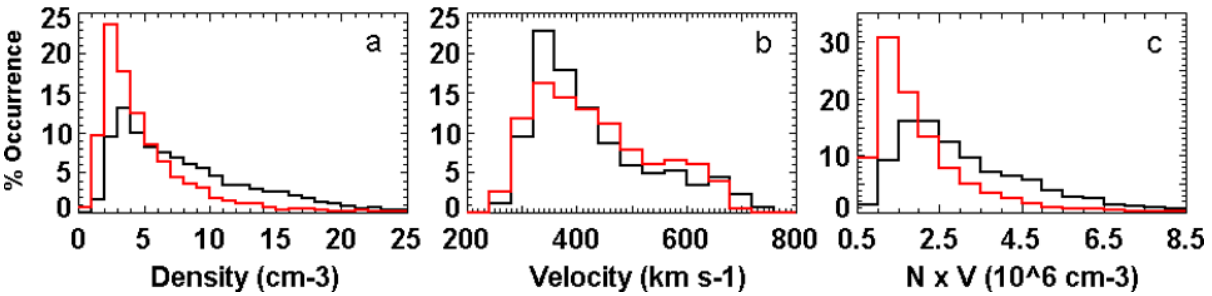

Figure 3 Histogram of occurrence at $1 \mathrm{AU}$ for a solar minimum period spanning $10 \mathrm{CRs}$ (see text for specific CR ranges). The colors represent data from SC 23 (red) and SC 22 (black). Shown are histograms for (a) density, (b) velocity, and (c) momentum flux, $N \times V$.

Figure 4 (a) Histogram of occurrence for the magnetic field magnitude observed over $10 \mathrm{CRs}$ during SC 22 (black) and 23 (red). (b) Similar histogram for STEREO-A (dark blue) and STEREO-B (light blue) observations overplotted with SC 23 (red)
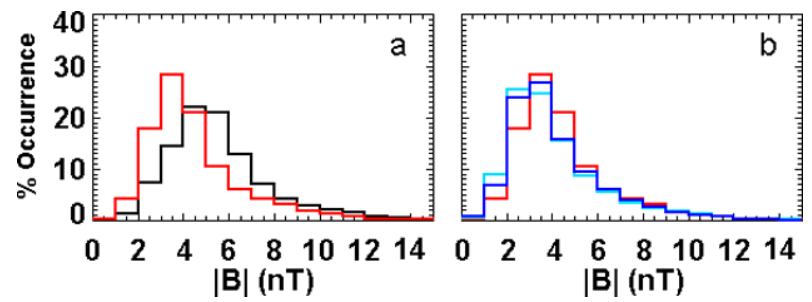

Figure 3(b) shows a similar histogram but for velocity. Both the SC 22 and 23 velocity distributions have peak values occurring around $340 \mathrm{~km} \mathrm{~s}^{-1}$. Notice that for SC 22 the percent occurrence of this peak distribution is $40 \%$ greater than that for SC 23 . For both periods a high-speed tail distribution can be seen centered near $580 \mathrm{~km} \mathrm{~s}^{-1}$, although the percent occurrence is slightly larger for SC 23. Figure 3(c) shows the momentum flux $(N \times V)$ for the two solar cycle periods. For SC 23, the peak occurs around $1.25 \mathrm{~cm}^{-2} \mathrm{~s}^{-1}$, which is about $38 \%$ less than the peak value for the SC 22 period $\left(2 \mathrm{~cm}^{-2} \mathrm{~s}^{-1}\right)$. Note that the decrease in the momentum flux during SC 23 is controlled by the density (see Figure 3(a)). This lower momentum flux is consistent with recent findings by McComas et al. (2008) using Ulysses data from high heliolatitudes, although as will be discussed in the following, the source(s) differ.

Figure 4(a) shows a histogram of the magnetic field magnitude. There is an overall shift toward lower values in the distribution of the field magnitude during SC 23 in comparison with SC 22. The peak of the distribution for the SC 23 period is centered at $3.5 \mathrm{nT}$, which is $30 \%$ less than $5 \mathrm{nT}$, the approximate central value for the peak of the SC 22 distribution. Figure 4(b) shows a comparison of the OMNI SC 23 observations with the 10-minuteresolution STEREO-A and STEREO-B magnetometer data (Acuña et al., 2008) obtained from the STEREO in situ data Web site (http://www-ssc.igpp.ucla.edu/ssc/stereo/) hosted by the Institute of Geophysics and Planetary Physics at the University of California, Los Angeles (e.g., Luhmann et al., 2008). The STEREO observations exhibit the same lower field distribution as the OMNI data.

To illustrate how the individual IMF components are contributing to the overall lower field magnitude during this current solar minimum period, we plot histograms of the standard RTN components. Figure 5(a) shows the histogram for the absolute values of the radial field. The SC 23 distribution is shifted toward lower field values, with the peak occurrence centered at $1.5 \mathrm{nT}$. The SC 22 distribution is slightly broader and has the peak occurrence centered at $2.5 \mathrm{nT}$. For the SC 23 period, radial field values that are $\leq 3 \mathrm{nT}$ occur more often, by $\approx 25 \%$, than during the SC 22 period. Figure $5(\mathrm{~b})$ shows a similar histogram, but for 

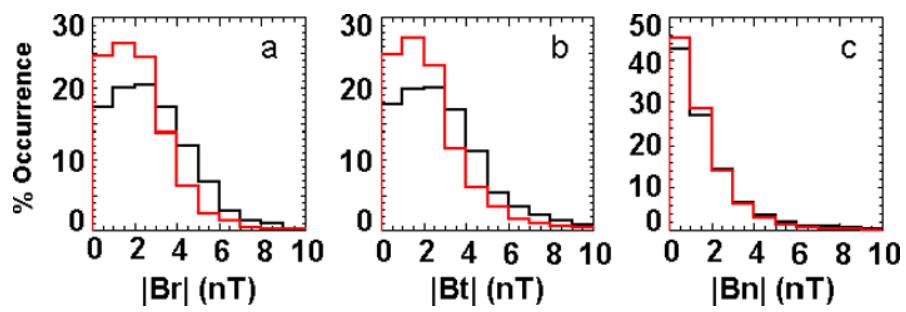

Figure 5 Histogram of occurrence for the absolute values of the (a) radial (R), (b) tangential (T), and (c) normal (N) magnetic field components observed over 10 CRs during SC 22 (black) and 23 (red).
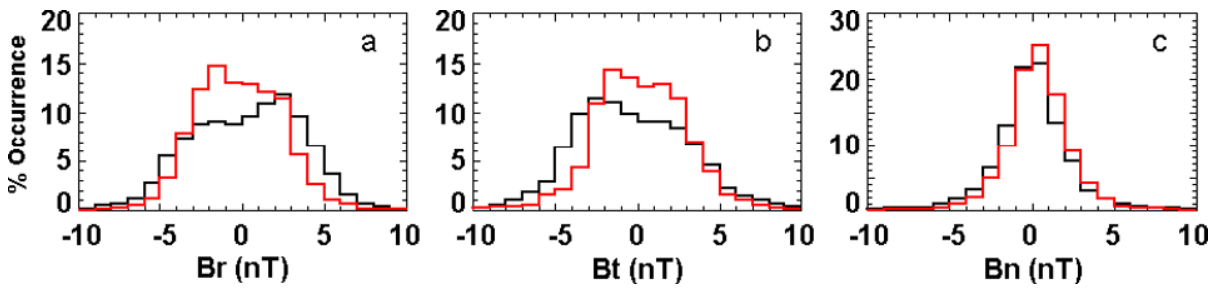

Figure 6 Histogram of occurrence for the (a) radial (R), (b) tangential (T), and (c) normal (N) magnetic field components observed over 10 CRs during SC 22 (black) and 23 (red).

the absolute values of the tangential field. The features shown are very similar to what was described previously for the radial fields in Figure 5(a). Figure 5(c) shows the histogram for the absolute values of the normal field. In this case, the distributions for SC 22 and 23 share a similar trend and overlap with one another.

Figure 6 shows histograms of the same data as in Figure 5 but with sign information preserved. The asymmetry around zero of the radial (Figure 6(a)) and tangential (Figure 6(b)) components for the SC 23 period is an indication of the recently observed unbalanced ecliptic plane magnetic field polarity. Such unbalanced field polarities are observed quite commonly (e.g., Hiltula and Mursula, 2006) and can be envisioned as a signature of greater heliospheric divergence of a particular sign of the coronal open fields. Figure 6(a) shows that the asymmetry in the distribution of the radial field values switched between SC 22 and 23. When the fields switched polarity between the two solar cycles, the heliospheric current sheet maintained its southward cone shape; that is, the solar ballerina remained "bashful" (see Hiltula and Mursula, 2006, for a discussion).

\section{Association of the Low IMF with the Stream Structure}

Since stream structures are prominent during the solar minimum phase, we investigate how they differ during the two solar minimum periods described in this paper and consider the role(s) of their solar sources. Figure 7 shows the time series for velocity, density, and field magnitude observed during the SC 23 period. One can see that the high-speed streams (top panel) and their related high-density ridges (middle panel) are common and well formed for this period. The red data points are data that have been filtered for field magnitudes $\leq 4 \mathrm{nT}$ (bottom panel). The filtered data periods are then plotted over the entire data set including the density and velocity data. There is a clear association of the low field magnitudes with the trailing part of the high-speed streams, where the rarefaction regions occur behind the 

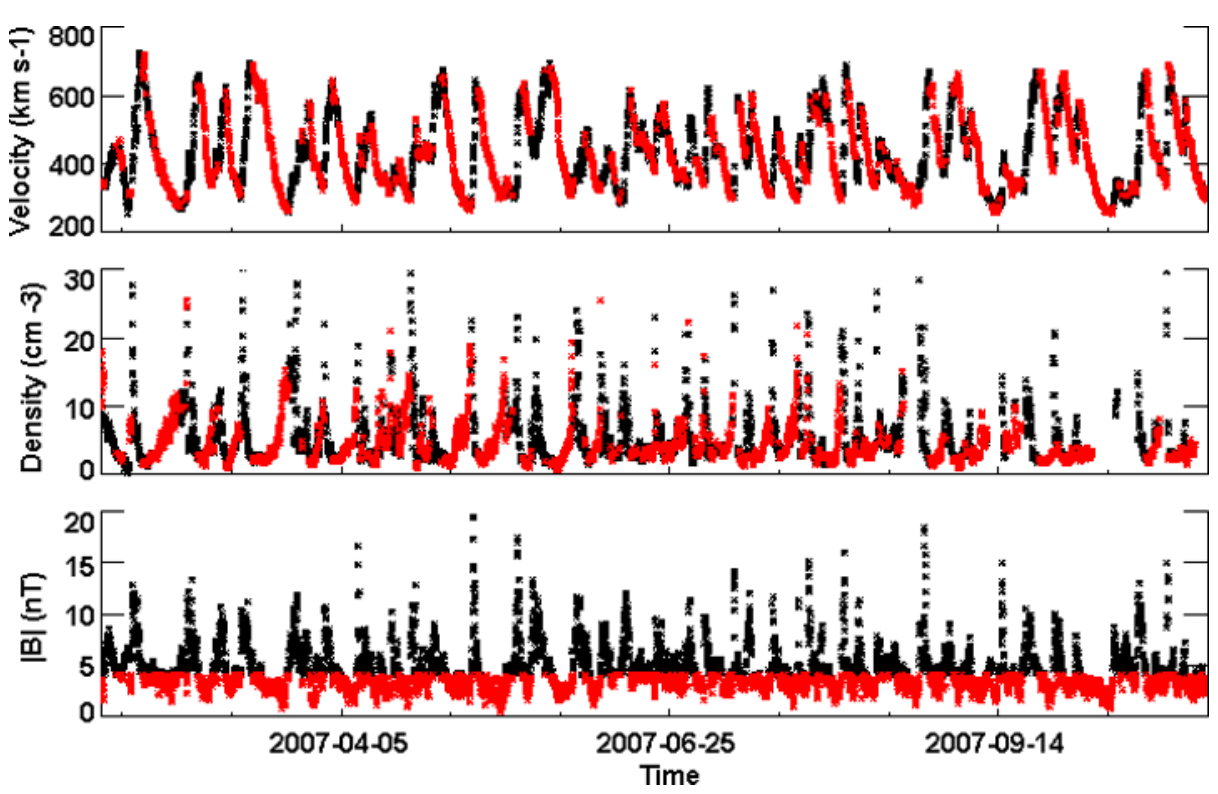

Figure 7 (Top to bottom) Time series of the velocity, density, and field magnitude for the SC 23 time period shown in Figures 3 and 4 . The red values are data filtered for field magnitude values that are $\leq 4 \mathrm{nT}$.

related compression ridges. The association with the rise of the high-density ridges is also very pronounced.

Figure 8 shows a similar set of time series for the SC 22 period. In comparison with the SC 23 period shown in Figure 7, the top panel of Figure 8 shows that there are generally fewer high-speed streams during SC 22. As before, the red dots correspond to data that have been filtered for field magnitudes $\leq 4 \mathrm{nT}$. The association of field magnitudes that are $\leq 4 \mathrm{nT}$ with the trailing part of the high-speed streams is not as clean when compared to the current cycle (Figure 7, top panel). However, if we include data filtered for field magnitudes between 4 and $5 \mathrm{nT}$ (shown in blue), the quality of the association is similar to what is shown for SC 23.

To examine in more detail the correlation between the velocity structures and the field magnitudes observed at $1 \mathrm{AU}$ over the SC 22 and 23 periods, we plot time series that are organized by Carrington rotation and effectively stack them against each other to produce color contour plots, as shown in Figures 9 and 10. The $x$-axis displays the day of the CR (0 to 27), the $y$-axis displays the CR number (1893-1902 for SC 22 or 2053-2062 for SC 23), and the color represents the magnitude of the solar wind velocity (top panels) or total magnetic field (bottom panels). The black areas in the plots represent data gaps in the observations.

Figure 9 (top panel) shows the high-speed (red, orange) and low-speed (cyan, blue) stream structures, which were very prominent during the SC 23 period. For each Carrington rotation, there were typically two high-speed and corresponding low-speed streams. The bottom panel shows that the ridges of high magnetic field magnitudes (red) occur during the rise of the high-speed streams (top panel, regions where the colors abruptly transition from blue to red). In contrast, the ridges of low field magnitudes (blue) occur during the trailing part of the high-speed streams (top panel, regions where the colors slowly transition from yellow to green to blue). 

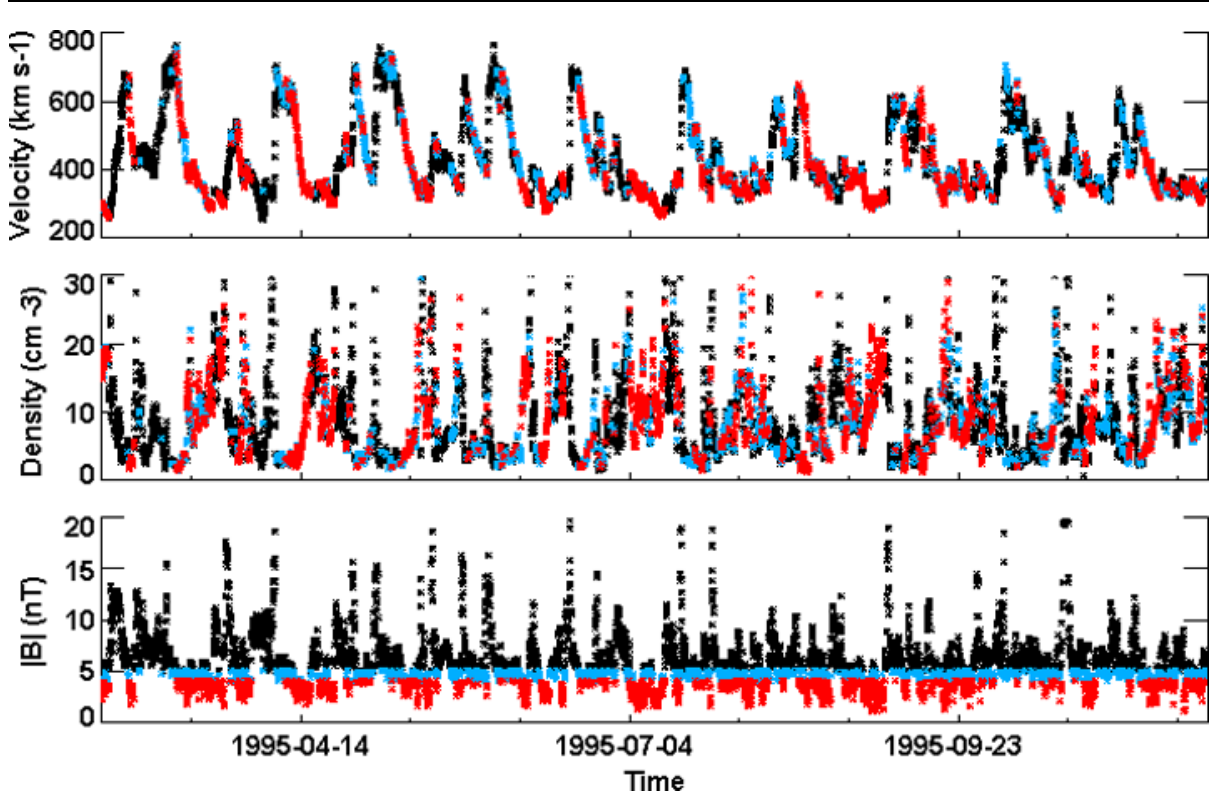

Figure 8 (Top to bottom) Time series of the velocity, density, and field magnitude for the SC 22 time period shown in Figures 3 and 4. The blue (red) values are data filtered for field magnitude values that are between 4 and $5 \mathrm{nT}(\leq 4 \mathrm{nT})$.

Figure 9 Color contour plot for the SC 23 time period. Shown are contours for (top panel) velocity $\left(\mathrm{km} \mathrm{s}^{-1}\right)$ and (bottom panel) magnetic field magnitude (nT).

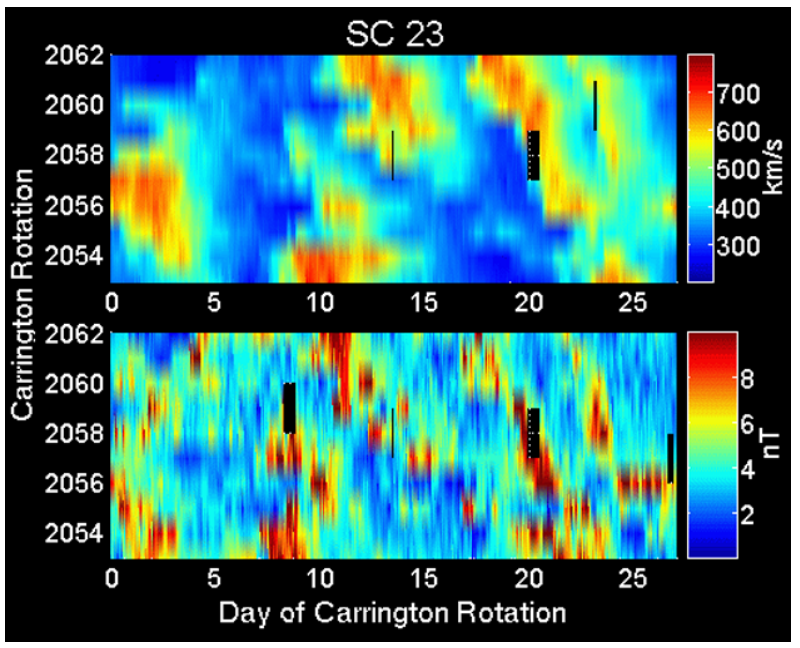

Figure 10 shows similar color contour plots, but for the SC 22 period. Although there are high- and low-speed stream structures during this time period (see top panel), the high-speed streams are not as abundant and well organized as those observed during the SC 23 period. Also, the contrast between the high- and low-speed stream structures (red to blue) is not as great. For example, the high-speed structure observed during CR 1896 on days 7 to 9 has a very gradual declining portion that lasts several days, where the speeds decrease from $\approx 550 \mathrm{~km} \mathrm{~s}^{-1}$ (yellow - green) to $\approx 400 \mathrm{~km} \mathrm{~s}^{-1}$ (cyan) before the next 
Figure 10 Color contour plot for the SC 22 time period. Shown are contours for (top panel) velocity $\left(\mathrm{km} \mathrm{s}^{-1}\right)$ and (bottom panel) magnetic field magnitude (nT).

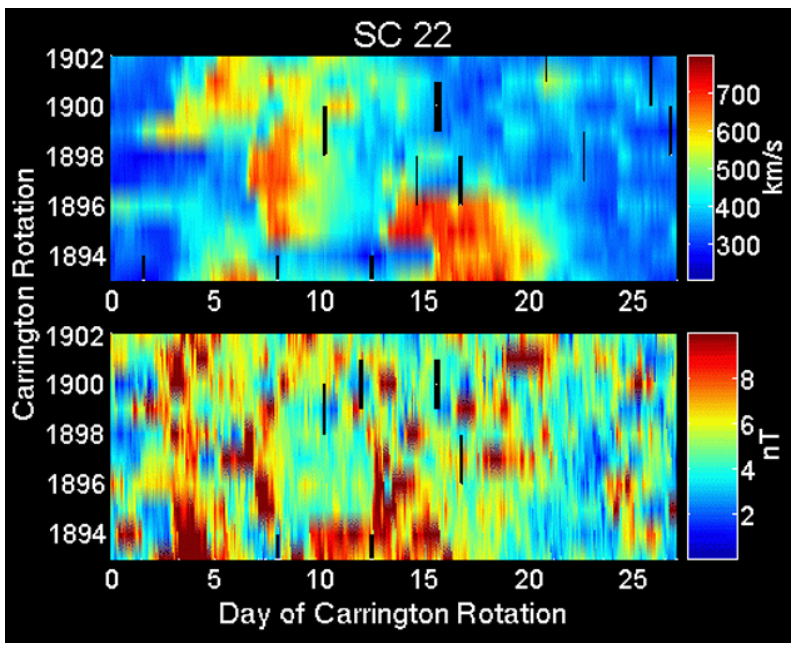

high-speed stream structure commences on days 13 to 18. In contrast, the trailing part of the high-speed streams observed in the SC 23 period shows a more abrupt decline. The speeds go from $\approx 550 \mathrm{~km} \mathrm{~s}^{-1}$ (yellow-green) to $\approx 400 \mathrm{~km} \mathrm{~s}^{-1}$ in a day or so, followed by a sharp transition to speeds below $350 \mathrm{~km} \mathrm{~s}^{-1}$ (blue), which lasts for several days before the next high-speed stream onset. Figure 10 (bottom panel) shows the magnetic field magnitude observed at this time. Notice that during SC 22 the field magnitudes have more mid-range (green to yellow) and high (red) field values and fewer low field values (blue) when compared to the current cycle. In addition, the ridges of high magnetic field magnitudes (red) do not necessarily occur during the onset of the high-speed streams, nor do the low field values always occur during the trailing part of the high-speed streams. We note that some solar transient events occurred during the time periods presented. The number of interplanetary coronal mass ejections (ICMEs) observed during the SC 22 period is eight compared to two during the SC 23 time period (see Jian et al., 2006, and http://www-ssc.igpp.ucla.edu/forms/stereo/stereo_level_3.html). This may in part explain the less organized nature of the SC 22 period.

We also examine the correlation between the observed densities and field magnitudes. Figure 11 shows color contours of the statistics of the magnetic field magnitudes versus density. The maximum values in the distribution are shown in red. For the SC 22 distribution, Figure 11(a) shows that the maximum occurrence ranges between field magnitudes of around 4-6 nT and density values of around 2-6 $\mathrm{cm}^{-3}$. For the SC 23 distribution (Figure 11(b)), the maximum occurrence distribution is shifted toward lower values in field magnitude and broader in range, from approximately 2 to $5.5 \mathrm{nT}$. The density distribution is shifted toward lower values as well (about $1.5-6 \mathrm{~cm}^{-3}$ ). Overall, the recent SC 23 period exhibits both lower field magnitudes and densities, consistent with the conclusions derived from Figures 5 through 10.

\section{Origin of the Low IMF}

To determine the nature of the differences in the present cycle interplanetary fields and their possible relationship to the weak solar polar fields mentioned in Section 1, it is necessary to consider their origins. Although we do not show this, from the computed 


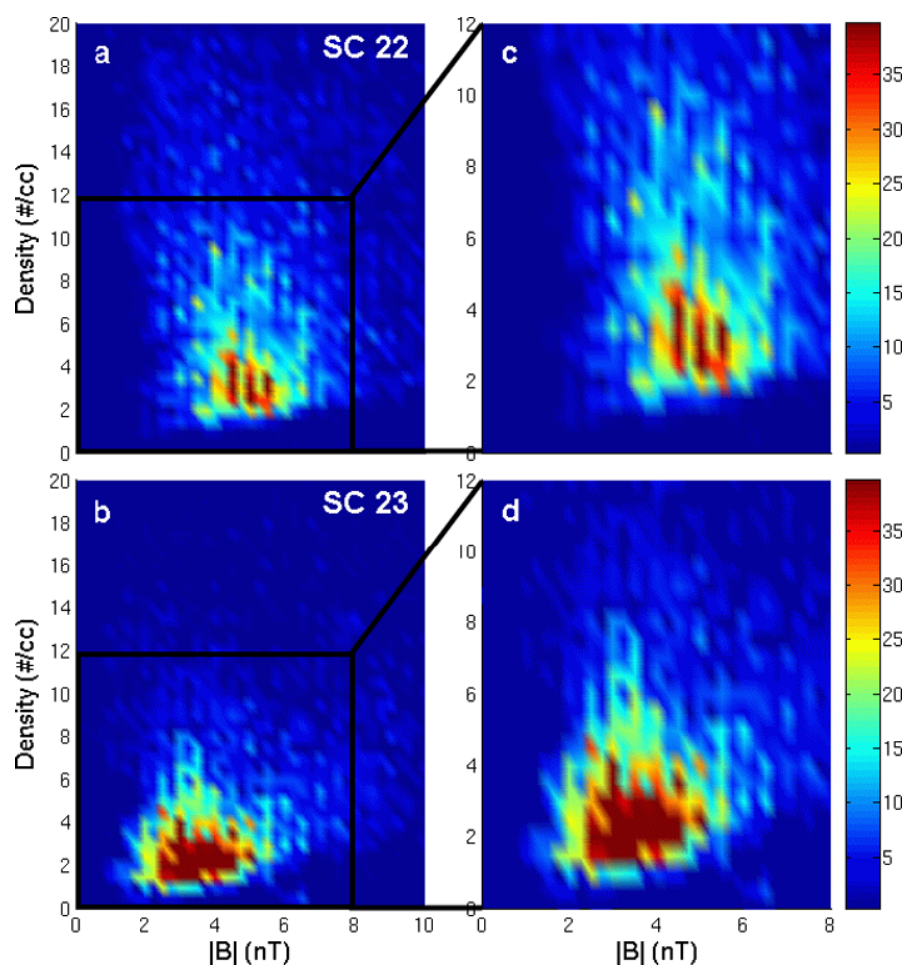

Figure 11 Color contour plots of the magnetic field magnitude versus density for (a) SC 22 and (b) SC 23; (c) zoomed-in version of (a) and (d) zoomed-in version of (b).

source surface coronal field maps available at the Wilcox Solar Observatory (WSO; http://wso.stanford.edu/synsourcel.html) the neutral line of the heliospheric current sheet (HCS) is seen to be more warped during CRs 2053-2062 in SC 23 in comparison with CRs $1893-1902$ in SC 22. If the slow wind belt roughly follows the neutral line, the inference is that the stream structure should be more pronounced for the solar cycle minimum in SC 23, which we do observe, as shown in Figure 7 (top panel). The greater warping should maximize the interaction between the high- and low-speed streams. In comparison, when the HCS is flat and near equatorial, as it is during the previous solar minimum, the stream interactions are less well formed and the ecliptic intersection of them shows less contrast. In particular, the formation of rarefactions in the ecliptic is expected to be weaker, as suggested by the results of Riley and Gosling (2007).

The sources of the high-speed streams are also different for these two near-solar minimum periods. Using the Wang - Sheeley - Arge (WSA) semiempirical solar wind model (Arge et al., 2004), we locate the sources of the high-speed streams observed at 1 AU. Standard plots used to map the solar wind sources with the WSA model are shown in Figures 12 and 13 for several Carrington rotations from the periods under study. Here, the colored regions are the predicted footpoints of the open field lines at the photosphere (i.e., the coronal holes), where the colors indicate the solar wind speed at $21.5 R_{\odot}$ arising from a particular open field region, and the black lines indicate where the open regions are magnetically connected to the ecliptic plane. Figure 12 suggests that for the time period in SC 22 , the sources of the ecliptic solar wind streams are mostly polar coronal hole edges and 

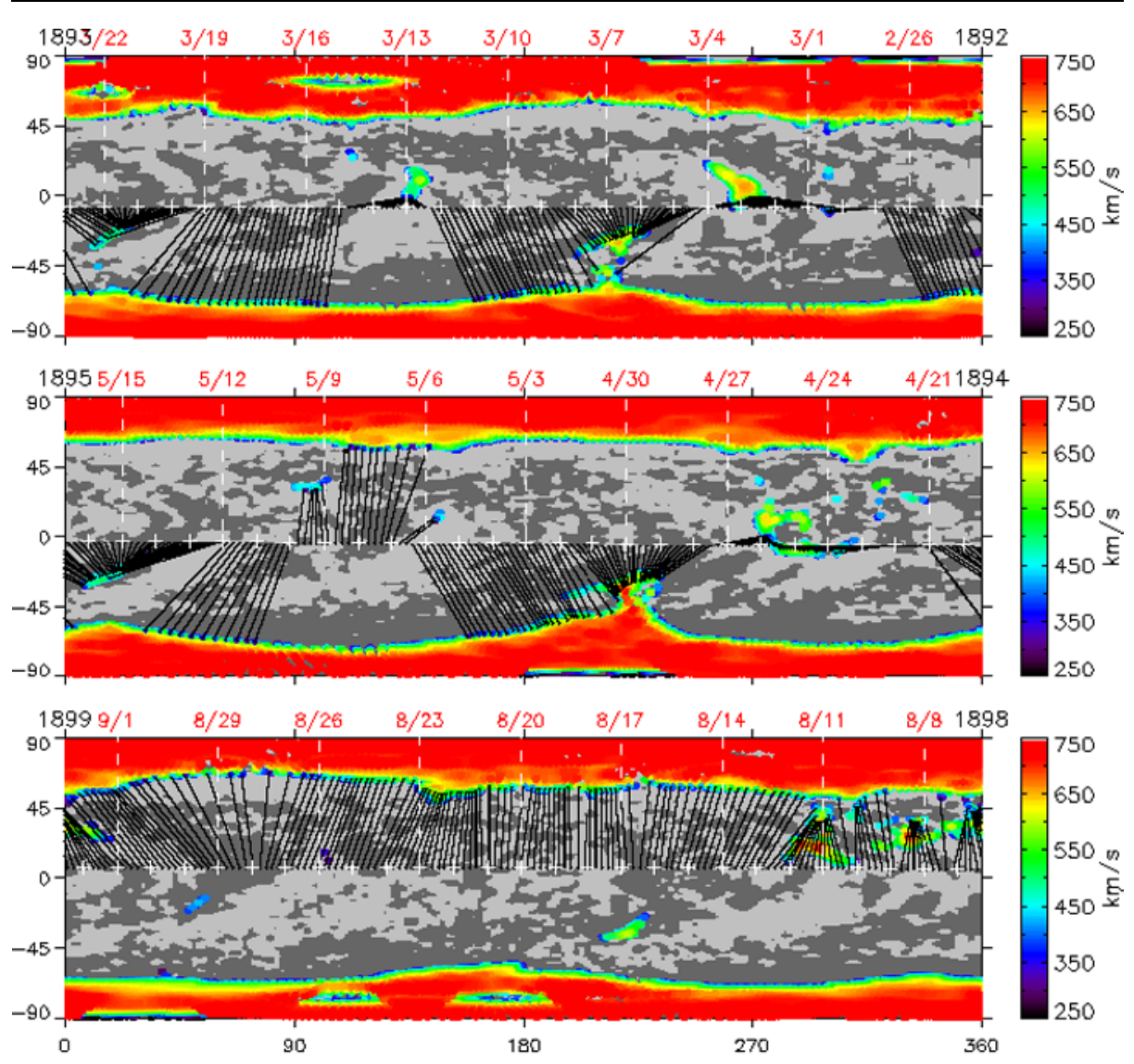

Carrington Longitude

Figure 12 The derived coronal sources for CRs 1893 (top), 1895 (middle), and 1899 (bottom) using the WSA coronal model. The calculated footpoints (colored dots) of the open field lines at the photosphere are shown. The different colors indicate the solar wind speed (at $21 R_{\odot}$ ) associated with the flux tubes, whereas the solid black lines connect the outer coronal boundary at $21.5 R_{\odot}$ and the source regions of the solar wind at the photosphere. The + symbol indicates the daily position of the sub-Earth point on the Sun, which varies between $\pm 7.25^{\circ}$ in solar latitude owing to the inclination of solar rotation axis with respect to the ecliptic plane. Note that the newest (oldest) data are located on the left side (right side).

extensions. In contrast, Figure 13 shows that, for SC 23, most of the high-speed streams originate from isolated open field sources located in the low- and mid-latitude regions. It seems that there were very few comparable low- and mid-latitude holes supplying the highspeed wind during the SC 22 period. Extreme-ultraviolet (EUV) images from the Solar and Heliospheric Observatory (SOHO) Extreme ultraviolet Imaging Telescope (EIT) (Delaboudiniere et al., 1995) and STEREO Sun Earth Connection Coronal and Heliospheric Investigation (SECCHI) Extreme UltraViolet Imager (EUVI) (Howard et al., 2002) confirm this difference.

To investigate the shapes and heliospheric extent of the high-speed stream sources, we consider the global outward mapping of the various coronal holes that are supplying the solar wind during the SC 22 and 23 periods. We use the potential field source surface (PFSS) approximation of the coronal field ( $c f$. Schatten, Wilcox, and Ness, 1969) in the same manner 

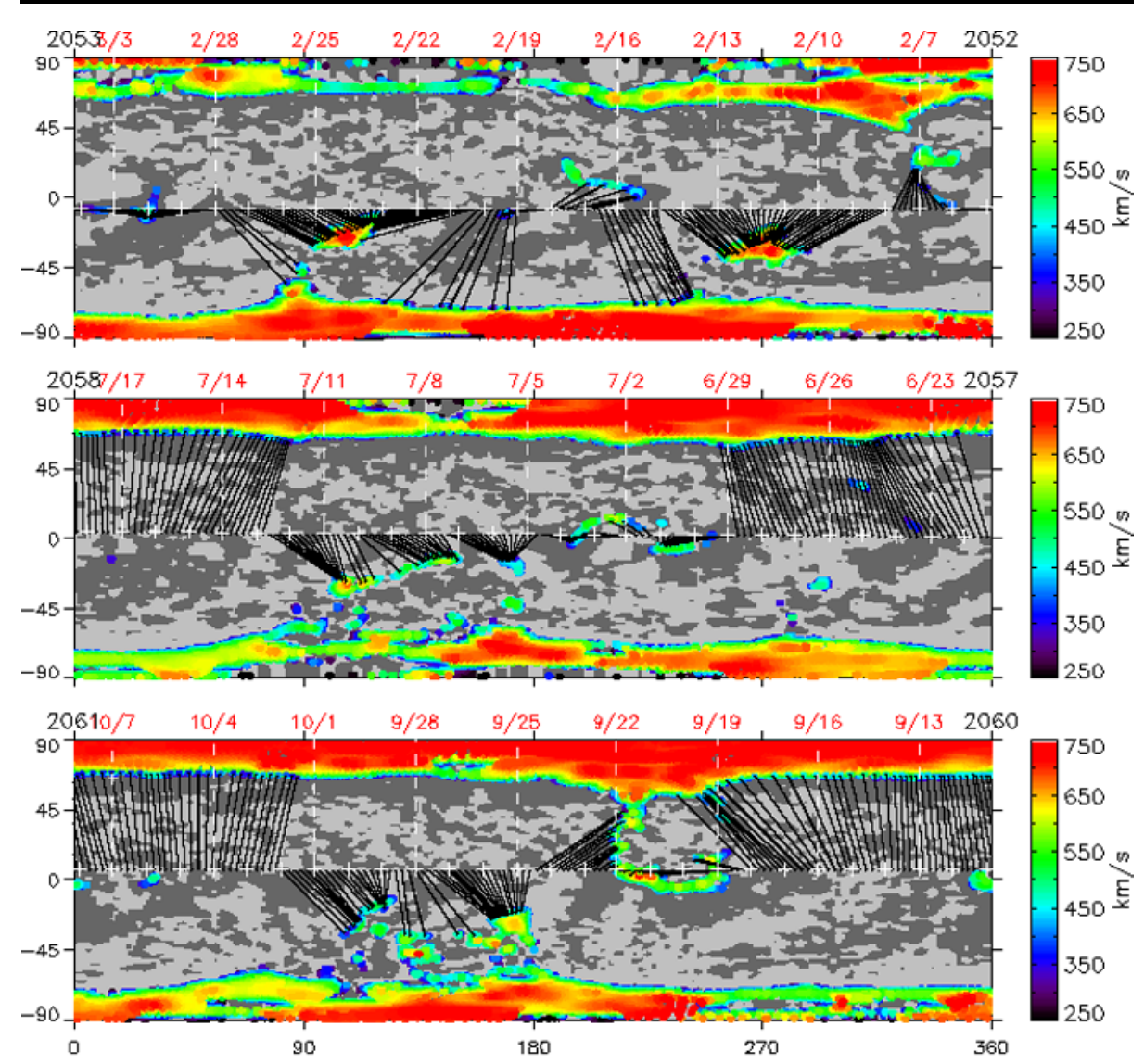

Carrington Longitude

Figure 13 The derived coronal sources for CRs 2053 (top), 2058 (middle), and 2061 (bottom) using the WSA coronal model.

as Zhao and Webb (2003) to map each derived open field region from the photosphere out to the source surface at $2.5 R_{\odot}$ (solar radii). Magnetograms from the WSO are used as input to the PFSS model.

Figure 14 (top panel) shows the open and closed field regions below $1.25 R_{\odot}$ for CR 1898 during the SC 22 period. The solid black curve marks the magnetic neutral line, whereas the color dotted areas and the blue - red field lines indicate the open and closed field regions, respectively. Six regions of open field lines can be seen for CR 1898, as shown in different colors. Three regions are polar coronal hole $(\mathrm{PCH})$ extensions, two are polar coronal holes, and one is an isolated low- to mid-latitude coronal hole. The second panel shows the various photospheric sources mapped to the source surface. Note that the colors correspond to the colored regions shown in the top panel. The magnetic polarities of the open field regions are indicated by the plus and minus symbols for open field pointing away from and toward the Sun, respectively. For CR 1898, the four isolated and PCH extensions of open field regions map out to a moderate latitude range at the source surface. Dominating the source surface map are open fields extending from within the $\mathrm{PCH}$ regions. The latitudinal extent of these open fields is wide, where some of the open fields map down to latitudes over 

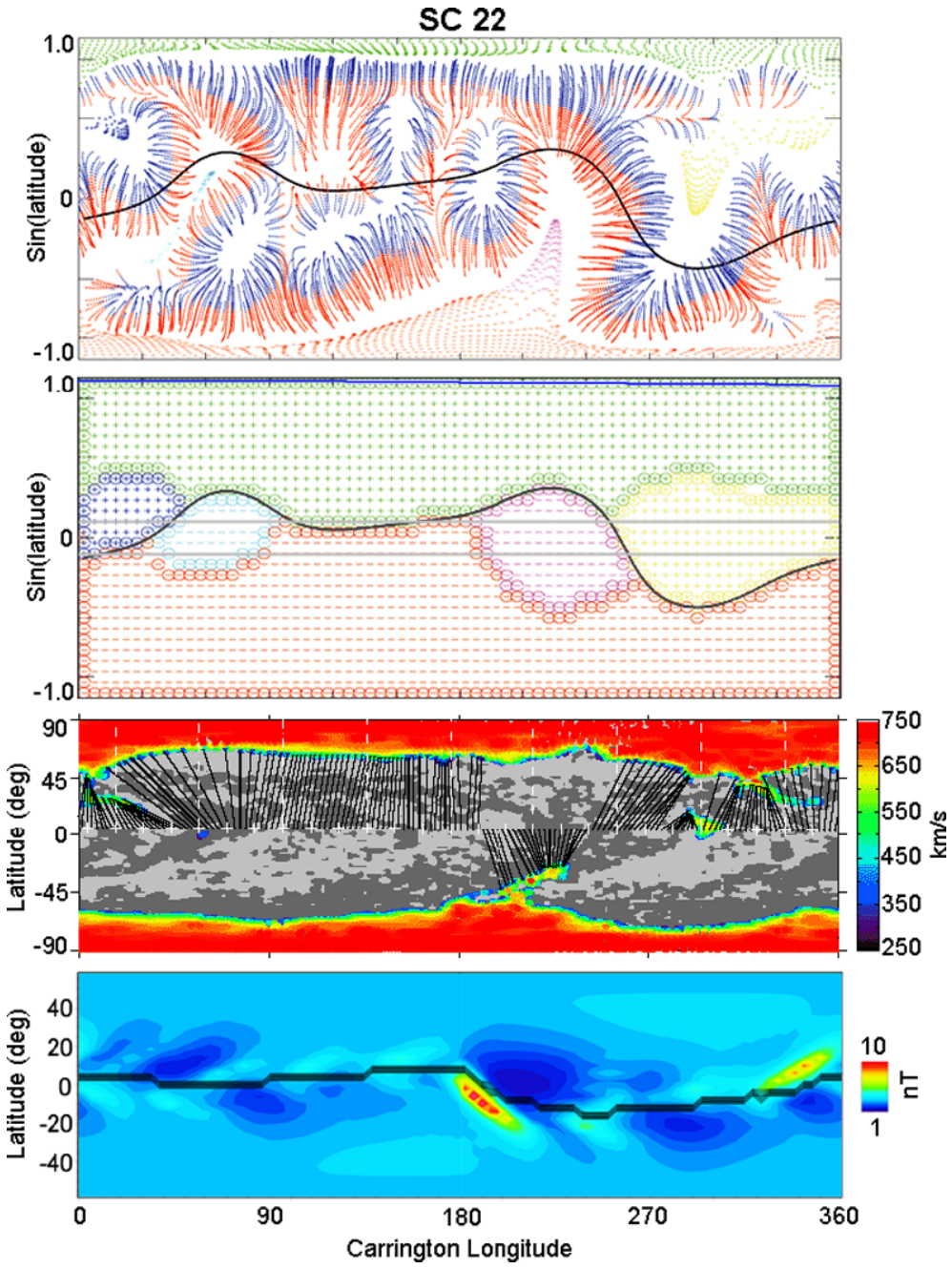

Figure 14 Global outward mapping of coronal holes supplying the solar wind for CR 1898 during the SC 22 period. (Top panel) Open (color dotted areas) and closed (areas with blue - red field lines) field regions below $1.25 R_{\odot}$. (Second panel) The photospheric sources mapped out to the source surface at $2.5 R_{\odot}$. The two solid gray bars mark the ecliptic plane location (between $\pm 7.25^{\circ}$ latitudes). The thick blue curve near the top of the panel marks the projected Ulysses trajectory. (Third panel) The WSA model predictions of the coronal hole areas and the solar wind speeds arising from them. (Bottom panel) The magnetic field magnitude at $1 \mathrm{AU}$ modeled by the coupled MAS/ENLIL solar wind model. The black curve seen throughout is the magnetic neutral line.

a broad longitude range within the ecliptic plane (between $\pm 7.25^{\circ}$ latitudes) as indicated by the two solid gray bars. Other Carrington rotations from the SC 22 period of our study also show similar features, where the open fields from the PCHs have very wide latitudinal extents at the source surface and the isolated and $\mathrm{PCH}$-extension regions of open fields have a moderate to narrow latitudinal range. This is consistent with the Ulysses results of McComas et al. (2008), who showed that the band of solar wind variability was narrow and confined to low latitudes during this period. The solid blue curve near the top of this 
second panel marks the projected Ulysses trajectory for this Carrington rotation (data is from http://cohoweb.gsfc.nasa.gov/helios/heli.html).

The third panel of Figure 14, similar to Figures 12 and 13, shows the WSA model predictions of the coronal hole areas and the solar wind speeds arising from them. The crosses mark the daily position of the sub-Earth point on the Sun, providing information about where the solar wind observed at Earth is coming from on the Sun. For CR 1898, the majority of the medium- to high-speed winds in the ecliptic are coming from the $\mathrm{PCH}$ edges and extensions. Noteworthy are subtle differences between the top and third panels with regard to the open field areas, in terms of shapes, locations, etc. These differences arise because the PFSS model here uses magnetograms from WSO whereas the WSA model uses magnetograms from the National Solar Observatory (NSO) (Henney, Keller, and Harvey, 2006) and a coupled coronal model consisting of the PFSS and Schatten current sheet model (Schatten, 1971). However, these differences do not affect the basic information obtained from these source mappings.

The bottom panel of Figure 14 shows the mapping of the magnetic field magnitude at 1 AU based on the open field regions shown for CR 1898. The results are generated by the coupled coronal MHD Around a Sphere (MAS) model (Riley, Linker, and Mikic, 2001), together with the ENLIL solar wind model (Odstrcil, 2003) (henceforth MAS/ENLIL). The coupled model uses NSO magnetograms and is publicly available through the runs-on-request service at the Community Coordinated Modeling Center (CCMC) (http://ccmc.gsfc.nasa.gov). The colors shown are the field magnitude values in the units of nanotesla, though it should be mentioned that the MAS/ENLIL solar wind model values tend to be underestimated by a factor of $\approx 2$ compared to observations (see Lee $e t$ $a l ., 2009$, for a discussion). The regions of low field magnitudes (patches of dark blue) are confined to a narrow latitudinal range and usually coincide with the rarefaction regions in the trailing parts of the high-speed streams (e.g., Figures 7 and 8), which in this case are confined to fairly low latitudes.

Figure 15 shows similar plots to Figure 14 but for CR 2060 during the SC 23 period. The top panel shows six isolated low- to mid-latitude coronal holes that map out to a much wider range of latitudes at the source surface (second panel) than for the SC 22 period shown previously. In addition, the open field regions from the $\mathrm{PCH}$ regions do not have as large a latitudinal extent over a broad range of longitudes, in contrast to Figure 14 (second panel), which is representative of the SC 22 period. Other Carrington rotations from the SC 23 period also show the low- to mid-latitude coronal holes mapping out to a much wider latitude range over a broad range of longitudes at the source surface. This is consistent with the Ulysses results of McComas et al. (2008), who also showed that the band of solar wind variability has a larger latitudinal extent for this period compared with the previous SC 22 period. The implication is that Ulysses would have spent more time during the SC 23 period in the solar wind emanating from the low- to mid-latitude sources than in the previous period. The solid blue curve shown in the second panel marks the Ulysses trajectory for this Carrington period, showing that Ulysses was crossing the ecliptic plane during this time. The third panel shows the WSA predictions of the coronal holes and the speeds arising from them. In the ecliptic plane, most of the high-speed winds were coming from these low- to mid-latitude sources of open fields, in contrast to the previous period where the majority of the high-speed winds came from the PCH edges. The bottom panel shows the MAS/ENLIL model results for the field magnitude at 1 AU. Regions with low field magnitude values are more abundant during this period and have a broader latitudinal range compared with the previous period.

The evolution of stream interactions and their related rarefactions may contribute to the weaker fields that are observed during this current minimum period. We investigate how the 

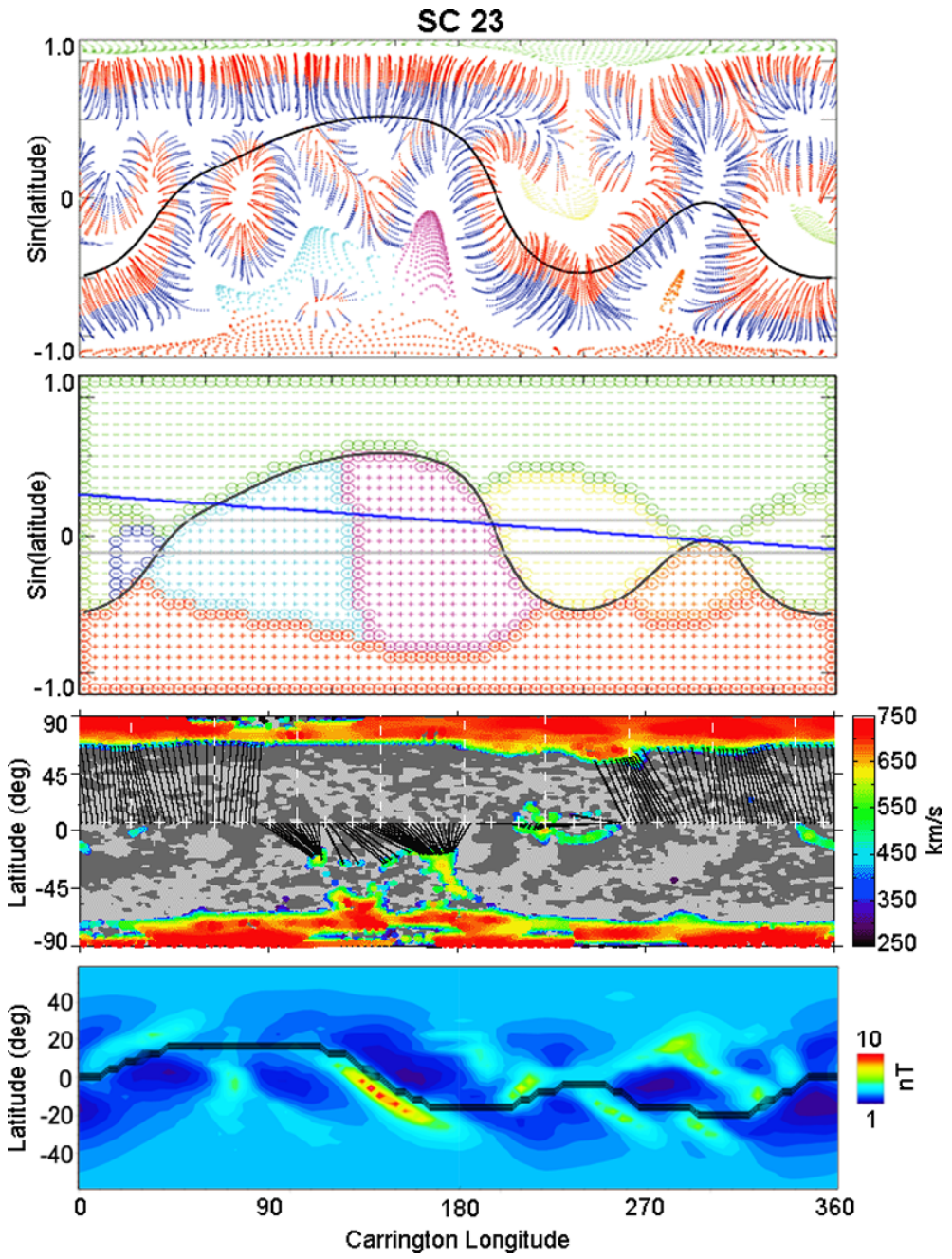

Figure 15 Global outward mapping of coronal holes supplying the solar wind for CR 2060 during the SC 23 period. (Top panel) The open (color dotted areas) and closed (areas with blue - red field lines) field regions below $1.25 R_{\odot}$. (Second panel) The photospheric sources mapped out to the source surface at $2.5 R_{\odot}$. The two solid gray bars mark the ecliptic plane location (between $\pm 7.25^{\circ}$ latitudes). The thick blue curve marks the projected Ulysses trajectory. (Third panel) The WSA model predictions of the coronal hole areas and the solar wind speeds arising from them. (Bottom panel) The magnetic field magnitude at 1 AU modeled by the coupled MAS/ENLIL solar wind model. The black curve seen throughout is the magnetic neutral line.

depth of the rarefactions, and thus the associated field magnitudes, depend on the low- and high-speed contrasts of the solar wind streams. Figures 16 and 17 illustrate the basic dynamical features of corotating compression and rarefaction regions in the equatorial plane (e.g., Pizzo, 1982; Riley, Linker, and Mikic, 2001). These numerical results are from the MAS coronal-solar wind model and were used by Riley and Gosling (2007) to study the origins of radial heliospheric fields in high-speed stream rarefaction regions. The initial conditions for the simulations consisted of a $300 \mathrm{~km} \mathrm{~s}^{-1}$ slow-speed stream throughout, with a single high-speed stream of either $400 \mathrm{~km} \mathrm{~s}^{-1}$ (Figure 16a) or $800 \mathrm{~km} \mathrm{~s}^{-1}$ (Figure 16b) from a 

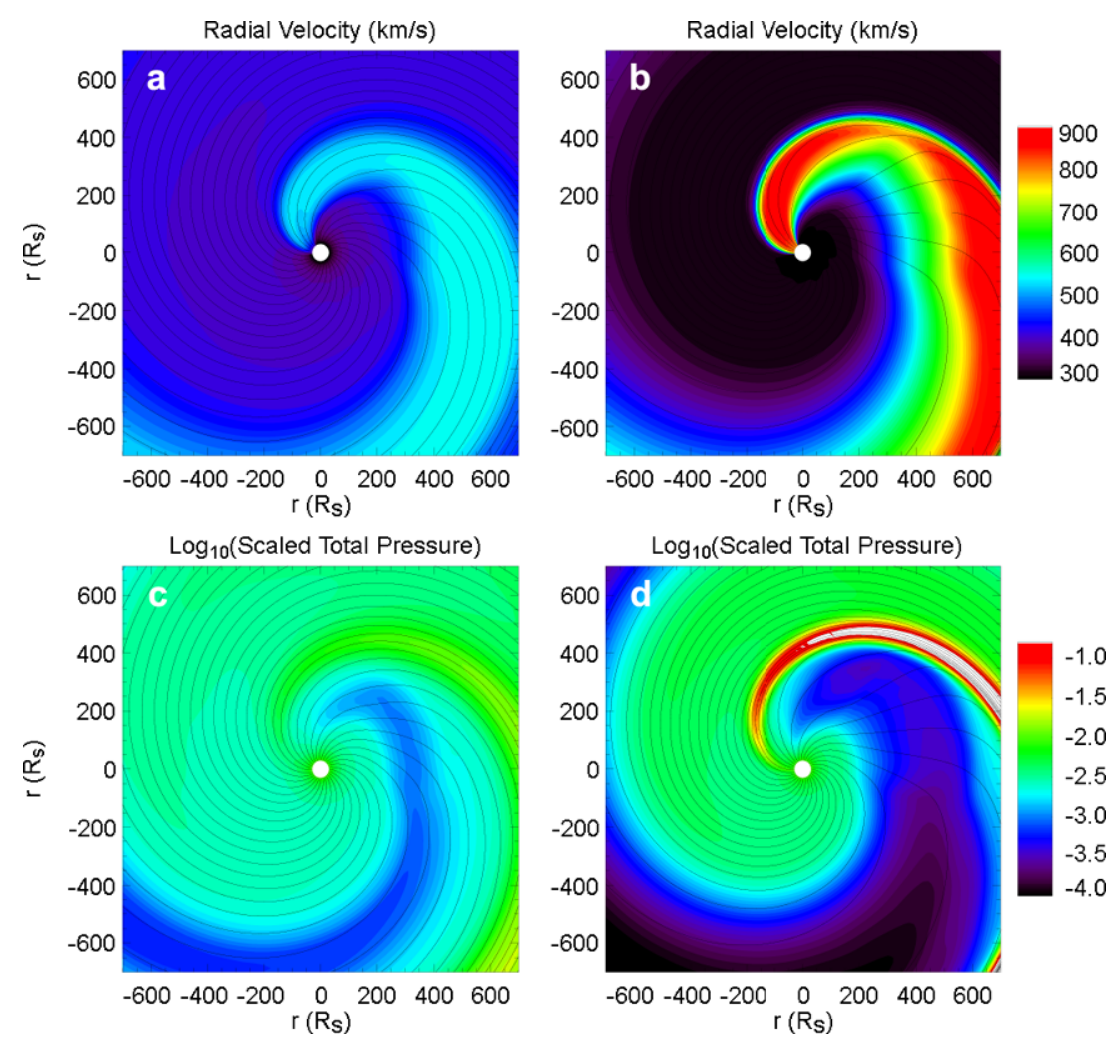

Figure 16 Numerical results generated by the MAS coronal-solar wind model in the equatorial plane for $(\mathrm{a}, \mathrm{b})$ radial velocity and (c, d) total pressure (magnetic and thermal, scaled for the adiabatic, spherical expansion of the solar wind). The initial conditions for the simulations consisted of a $300 \mathrm{~km} \mathrm{~s}^{-1}$ low-speed stream throughout, with a single high-speed stream of either $400 \mathrm{~km} \mathrm{~s}^{-1}$ (a, c) or $800 \mathrm{~km} \mathrm{~s}^{-1}$ (b, d). The $x$ and $y$-axes are given in solar radii, where $\sim 214 R_{\mathrm{S}}$ is equivalent to $1 \mathrm{AU}$.

coronal hole centered on the equator. At the boundary where the high-speed wind overtakes the slow-speed wind, a compression region forms where the pressure is higher and the field lines are closer together. For the slow-speed contrast case (Figure 16(c)), the compression region is much weaker (i.e., lower pressure and less compressed fields), compared to that for the high-speed contrast case (Figure 16(d)). Behind the compression region in both cases, a rarefaction region forms where the high-speed wind outruns the slow-speed wind behind it. However, for the high-speed contrast case, the rarefaction region has a much larger radial extent and is more rarefied. In addition, the ratio $B_{r} / B$ is larger, where the near-radial fields occupy a broader region in radial distance and longitude (compare Figures 17(b) with (a)). Figures 17(c) to (f) show the details of the magnetic field magnitude and radial field (scaled by $r$ and $r^{2}$, respectively). For the high-speed contrast case (Figures 17(d) and (f)), the magnetic field is noticeably lower behind the compression regions. These model results suggest that since the speed contrasts are significantly higher during the SC 23 period (see discussion for Figures 7 and 9), there will be deeper rarefaction regions and thus lower magnetic fields in those regions.

The MAS model result shown in Figure 17(b) demonstrates that streams with high-speed contrasts have stronger near-radial field regions. Since it is known that during periods of 

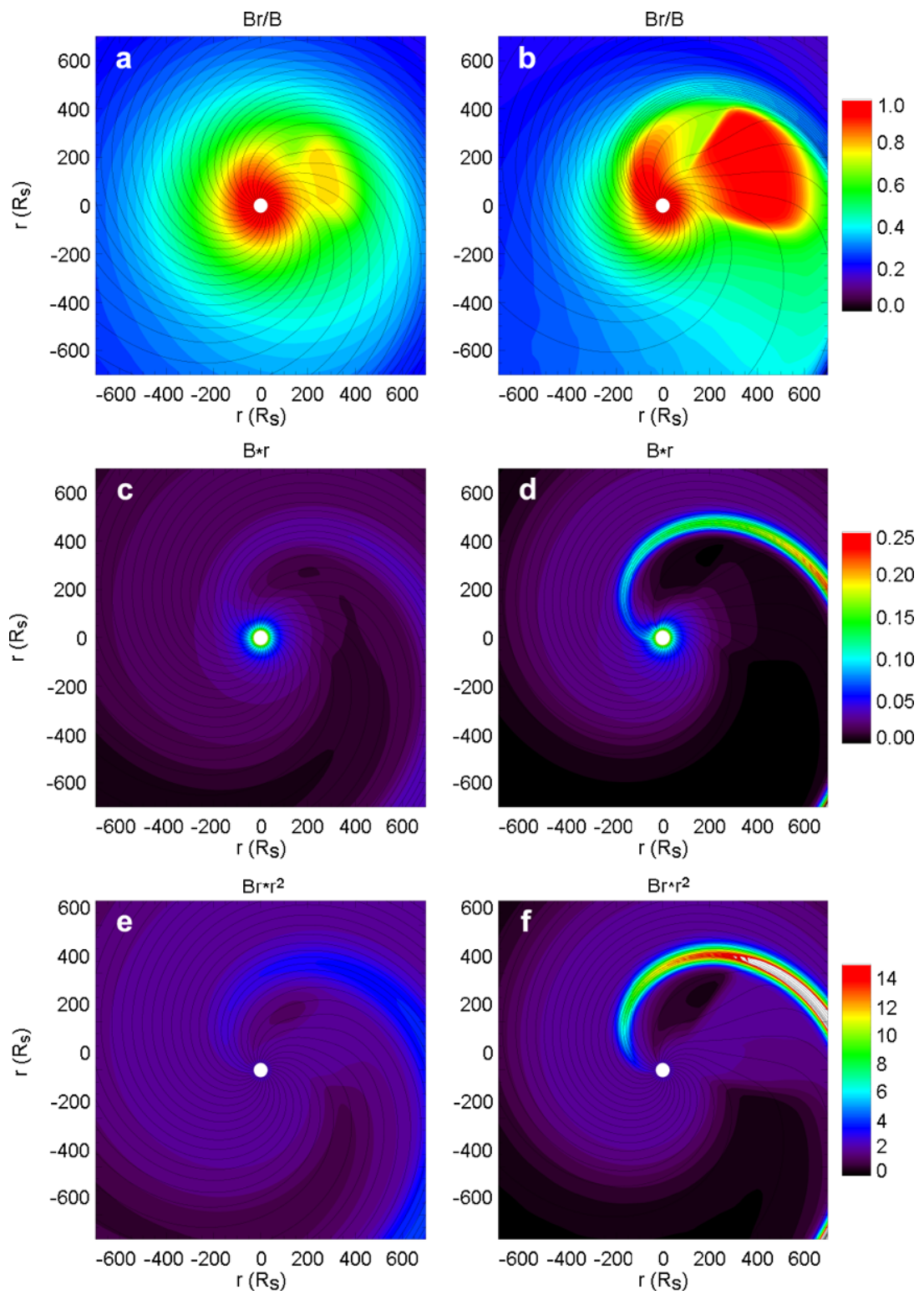

Figure 17 Numerical results for $(\mathrm{a}, \mathrm{b}) B_{r} / B,(\mathrm{c}, \mathrm{d})$ magnetic field magnitude scaled by $r$, and (e, f) radial magnetic field scaled by $r^{2}$.

near-radial fields, the field magnitudes and the densities are lower (Neugebauer, Goldstein, and Goldstein, 1997), we investigate the contribution of near-radial fields to the weaker fields observed during this solar cycle period. Figure 18 shows histograms of the OMNI data for the ratio $B_{r} / B$ (top row) and density (bottom row). The colors shown are for data that have been filtered for when the field magnitude is less than $4 \mathrm{nT}$ (blue) and greater than $4 \mathrm{nT}$ (magenta). During the SC 23 period (right column), there was an overall higher 

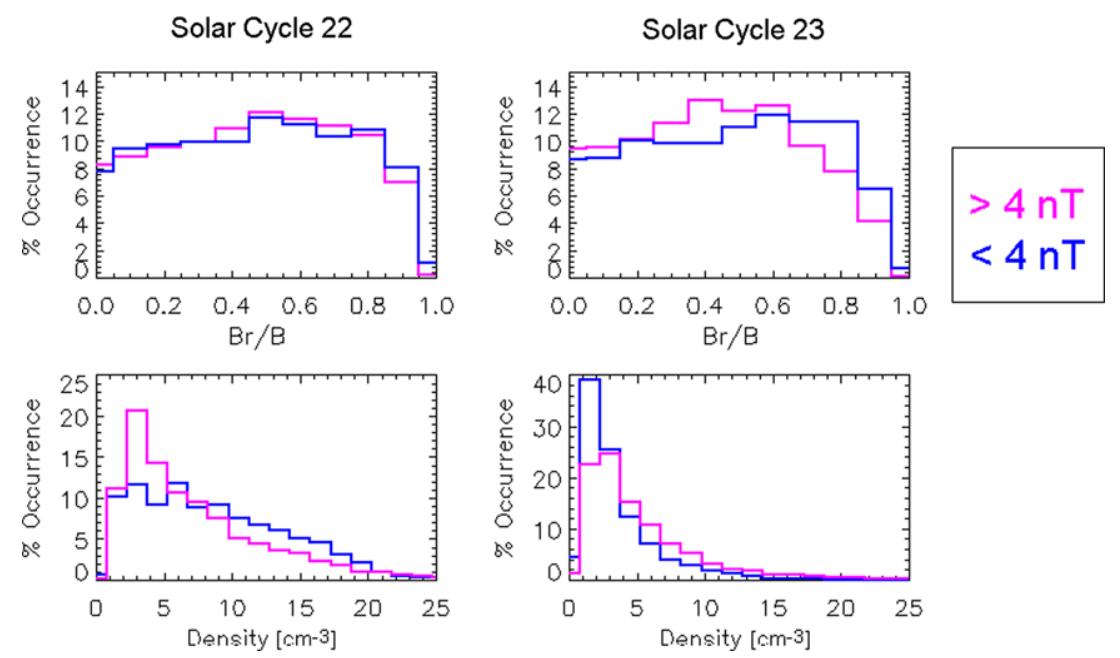

Figure 18 Histograms of occurrence at $1 \mathrm{AU}$ for (top row) the ratio $B_{r} / B$ and (bottom row) density. Data from the SC 22 (23) period are shown on the left (right). The colors shown are for data that have been filtered for when the field magnitude is less than $4 \mathrm{nT}$ (blue) and greater than $4 \mathrm{nT}$ (magenta).

fraction of the field magnitude that was more radial during the times of low field (less than $4 \mathrm{nT}$ ). For these low field periods, the fraction of low densities was also much higher. This was not the case for the SC 22 period, as shown in Figure 18 (left column). These OMNI results are consistent with the MAS model results shown in Figures 16 and 17 in that the SC 23 conditions, with stronger ecliptic sources of high-speed streams, seem to produce greater rarefactions with the associated more-radial fields.

\section{Summary and Discussion}

We investigated signatures of the unusually weak polar field in the ecliptic near-Earth IMF for the STEREO period of observations and compared the results with observations for a similar period from the previous solar cycle. Using the 1 AU OMNI data, for the SC 23 period we found the following for the peak of the distribution of values: Both the ecliptic IMF magnitude and density were 30\% lower, the momentum flux was $38 \%$ lower, and the velocity remained unchanged. This is consistent with the Ulysses off-ecliptic observations reported by McComas et al. (2008) and Smith and Balogh (2009). We showed that these weaker fields were clearly associated with the declining portions of the high-speed streams where the rarefaction regions occur.

Results from the WSA semiempirical solar wind model showed that sources of the highspeed streams were different for the SC 23 period than for the previous solar cycle, where the coronal holes were more isolated and resided in the low- to mid-latitude regions. The global modeling results from the PFSS model of the coronal open field regions suggested that these isolated low- to mid-latitude holes mapped out to a much greater latitudinal area of the heliosphere for SC 23 and dominated the ecliptic plane. The MAS/ENLIL global modeling results for $1 \mathrm{AU}$ showed that the regions of low field magnitude also have a broader longitudinal range and were more abundant during SC 23. Results from the MAS MHD coronal - solar wind model illustrated how the evolution of the stream interactions can contribute to weaker fields and suggested that, if the speed contrasts were higher during the 
SC 23 period, there would be deeper rarefaction regions and thus lower magnetic fields in those regions.

Based on the Ulysses observations reported by Smith and Balogh (2009), the weaker ecliptic fields may also be due to less open solar flux. However, since the ecliptic observations and model results shown in this study indicate that the low- to mid-latitude flux is not only from the polar regions (also see Luhmann et al., 2009, for a discussion) and is moreover latitudinally redistributed by stream interactions, the interpretation is complicated. Future modeling of both the Ulysses and ecliptic IMF data together (P. Riley, private communication, 2008) may shed more light on the origins of the weaker ecliptic IMF of this solar cycle period. In the meantime, caution must be exercised in evaluating these weaker ecliptic fields.

Acknowledgements The authors would like to thank Todd Hoeksema and Xu Dong Sun for their useful suggestions, the NASA Goddard Space Flight Center Space Physics Data Facility (SPDF) for providing the OMNI data access, the NASA/CCMC for their dedicated assistance in making the model computer runs needed for this study, and the National Solar Observatory and Wilcox Solar Observatory for providing access to their magnetogram data sets. This research was supported by the 2005-2006 National Defense Science and Engineering Graduate (NDSEG) Fellowship awarded by the Department of Defense (DoD) and the CISM project, which is funded by the STC Program of the National Science Foundation under agreement number ATM-0120950.

Open Access This article is distributed under the terms of the Creative Commons Attribution Noncommercial License which permits any noncommercial use, distribution, and reproduction in any medium, provided the original author(s) and source are credited.

\section{References}

Acuña, M.H., Curtis, D., Scheifele, J.L., Russell, C.T., Schroeder, P., Szabo, A., Luhmann, J.G.: 2008, The STEREO/IMPACT magnetic field experiment. Space Sci. Rev. 136, $203-226$.

Arge, C.N., Pizzo, V.J.: 2000, Improvement in the prediction of solar wind conditions using near-real time solar magnetic field updates. J. Geophys. Res. 105, $10465-10479$.

Arge, C.N., Luhmann, J.G., Odstrcil, D., Schrijver, C.J., Li, Y.: 2004, Stream structure and coronal sources of the solar wind during the May 12th, 1997 CME. J. Atmos. Solar Terr. Phys. 66, 1295- 1309.

Balogh, A., Beek, T.J., Forsyth, R.J., Hedgecock, P.C., Marquedant, R.J., Smith, E.J., Southwood, D.J., Tsurutani, B.T.: 1992, The magnetic field investigation on the Ulysses mission: Instrumentation and preliminary scientific results. Astron. Astrophys. Suppl. Ser. 92, $221-236$.

Bame, S.J., McComas, D.J., Barraclough, B.L., Phillips, J.L., Sofaly, K.J., Chavez, J.C., Goldstein, B.E., Sakurai, R.K.: 1992, The Ulysses solar wind plasma experiment. Astron. Astrophys. Suppl. Ser. 92, $237-265$.

Delaboudiniere, J.P., Artzner, G.E., Brunaud, J., Gabriel, A.H., Hochedez, J.F., Millier, F., Song, X.Y., Au, B., Dere, K.P., Howard, R.A., et al.: 1995, EIT: Extreme-ultraviolet imaging telescope for the SOHO mission. Solar Phys. 162, $291-312$.

Henney, C.J., Keller, C.U., Harvey, J.W.: 2006, SOLIS-VSM solar vector magnetograms. In: Casini, R., Lites, B.W. (eds.) Solar Polarization 4 CS-358, Astron. Soc. Pac., San Francisco, 92-95.

Hiltula, T., Mursula, K.: 2006, Long dance of the bashful ballerina. Geophys. Res. Lett. 33, L03105. doi:10.1029/2005GL025198.

Howard, R.A., Moses, J.D., Socker, D.G., Dere, K.P., Cook, J.W.: 2002, Sun Earth Connection Coronal and Heliospheric Investigation (SECCHI). Adv. Space Res. 29, 2017-2026.

Issautier, K., Le Chat, G., Meyer-Vernet, N., Moncuquet, M., Hoang, S., MacDowell, R.J., McComas, D.J.: 2008, Electron properties of high-speed solar wind from polar coronal holes obtained by Ulysses thermal noise spectroscopy: Not so dense, not so hot. Geophys. Res. Lett. 35, L19101. doi:10.1029/2008GL034912.

Jian, L., Russell, C.T., Luhmann, J.G., Skoug, R.M.: 2006, Properties of interplanetary coronal mass ejections at one AU during 1995-2004. Solar Phys. 239, 393-436.

Kaiser, M.: 2005, The STEREO mission: an overview. Adv. Space Res. 36, 1483 - 1488. 
Lee, C.O., Luhmann, J.G., Odstrcil, D., MacNeice, P.J., de Pater, I., Riley, P., Arge, C.N.: 2009, The Solar Wind at 1 AU During the declining phase of solar cycle 23: comparison of 3D numerical model results with observations. Solar Phys. 254, 155-183.

Luhmann, J.G., Li, Y., Arge, C.N., Gazis, P.R., Ulrich, R.: 2002, Solar cycle changes in coronal holes and space weather cycles. J. Geophys. Res. 107, 1154. doi:10.1029/2001JA007550.

Luhmann, J.G., Curtis, D.W., Schroeder, P., McCauley, J., Lin, R.P., Larson, D.E., Bale, S.D., Sauvaud, J.A., Aoustin, C., Mewaldt, R.A., et al.: 2008, STEREO IMPACT investigation goals, measurements, and data products overview. Space Sci. Rev. 136, $117-184$.

Luhmann, J.G., Lee, C.O., Li, Y., Arge, C.N., Galvin, A.B., Simunac, K., Russell, C.T., Howard, R.A., Petrie, G.: 2009, Solar wind sources in the late declining phase of cycle 23: effects of the weak solar polar field on high speed streams. Solar Phys. this issue. doi:10.1007/s11207-009-9354-5.

McComas, D.J., Ebert, R.W., Elliott, H.A., Goldstein, B.E., Gosling, J.T., Schwadron, N.A., Skoug, R.M.: 2008, Weaker solar wind from the polar coronal holes and the whole Sun. Geophys. Res. Lett. 35, 18103. doi:10.1029/2008GL034896.

Neugebauer, M., Goldstein, M., Goldstein, B.E.: 1997, Features observed in the trailing regions of interplanetary clouds from coronal mass ejections. J. Geophys. Res. 102, 19743-19751.

Odstrcil, D.: 2003, Modeling 3D solar wind structure. Adv. Space Res. 32, $497-506$.

Pizzo, V.J.: 1982, A three-dimensional model of corotating streams in the solar wind: magnetohydrodynamic streams. J. Geophys. Res. 87, 4374-4394.

Riley, P., Gosling, J.T.: 2007, On the origin of near-radial fields in the heliosphere: numerical simulations. J. Geophys. Res. 112, A07102. doi:10.1029/2006JA012210.

Riley, P., Linker, J.A., Mikic, Z.: 2001, An empirically-driven global MHD model of the solar corona and inner heliosphere. J. Geophys. Res. 106, 15889-15901.

Schatten, K.H.: 1971, Current sheet magnetic model for the solar corona. Cosm. Electrodyn. 2, 232 - 245.

Schatten, K.H., Wilcox, J.M., Ness, N.F.: 1969, A model of interplanetary and coronal magnetic fields. Solar Phys. 6, $442-455$.

Smith, E.J., Balogh, A.: 2009, Decrease in heliospheric magnetic flux in this solar minimum: recent Ulysses magnetic field observations. Geophys. Res. Lett. 35, in press. doi:10.1029/2008GL035345.

Svalgaard, L., Duvall Jr., T.L., Scherrer, P.H.: 1978, The strength of the Sun's polar fields. Solar Phys. 58, $225-240$.

Zhao, X.P., Webb, D.F.: 2003, Source regions and storm effectiveness of frontside full halo coronal mass ejections. J. Geophys. Res. 108, 1234. doi:10.1029/2002JA009606. 